\title{
An Experimental Study of Nonlinear Dynamic System Identification
}

\author{
Greselda I. Stry ${ }^{1}$ and D. Joseph Mook ${ }^{2}$
}

\author{
Department of Mechanical and Aerospace Engineering \\ State University of New York at Buffalo \\ Buffalo, New York 14260 \\ 716-636-3058
}

\begin{abstract}
A technique for robust identification of nonlinear dynamic systems is developed and illustrated using both digital simulations and analog experiments. The technique is based on the Minimum Model Error optimal estimation approach. A detailed literature review is included in which fundamental differences between the current approach and previous work is described. The most significant feature of the current work is the ability to identify nonlinear dynamic systems without prior assumptions regarding the form of the nonlinearities, in contrast to existing nonlinear identification approaches which usually require detailed assumptions of the nonlinearities. The example illustrations indicate that the method is robust with respect to prior ignorance of the model, and with respect to measurement noise, measurement frequency, and measurement record length.
\end{abstract}

\section{Introduction}

The widespread existence of nonlinear behavior in many dynamic systems is welldocumented, e.g. Thompson and Stewart [1]; Nayfeh and Mook [2]. In particular, virtually every problem associated with orbit estimation, flight trajectory estimation, spacecraft dynamics, etc., is known to exhibit nonlinear behavior. Many excellent methods for analyzing nonlinear system models have been developed. However, a key practical link is often overlooked, namely: How does one obtain an accurate mathematical model for the dynamics of a particular complicated nonlinear system?

Accurate dynamic models are necessary for analysis, filter design, and/or control system design. For example, most filter design assumes white process noise, yet many nonlinear effects are inherently non-zero mean; e.g., quadratic nonlinearities are always positive. In order to obtain a model with truly zero mean process noise for filter design

Graduate Resarch Assistant; NASA Graduate Researcher

Assistant Professor 
purposes, all of the quadratic terms (and many other nonlinearities) must be well modeled. However, the complexity of many real systems precludes the possibility of accurately constructing a dynamic model purely from analysis using the laws of physics.

Identification is the process of developing an accurate mathematical model for a system, given a set of output measurements. Much work has been done on identification of linear systems, resulting in a number of efficient algorithms. The accuracy and ease of application of these algorithms has given linear identification an enormous popularity. It is, therefore, a common practice to use linearized models to describe nonlinear systems. However, linearization does not work in every application, and even when it does provide a reasonable approximation, the approximation is normally limited to a small region about the operating point of linearization. Consequently, there is a real need for nonlinear identification algorithms. If nonlinearities are a predominant part of a system's behavior, using a linear model to describe such a system leads to inconsistencies ranging from inaccurate numerical results to misrepresentation of the system's qualitative behavior. Since nonlinearities are seldomly easily characterized, identification techniques may prove beneficial in developing accurate mathematical representations of nonlinear systems.

Numerous methods for the identification of nonlinear systems have been developed in the past two decades (Natke, Juang and Gawronski [3]). Most methods fall into one of the following categories:

1. describing the nonlinear system using a linear model

2. the direct equation approach

3. representing the nonlinear system in a series expansion, and obtaining the respective coefficients either by using a regression estimation technique, by minimizing a cost functional, by using correlation techniques, or by some other approach

4. obtaining a graphical representation of the nonlinear term(s), then finding an analytical model for the nonlinearity

With such diversity of nonlinear identification techniques, the choice of an algorithm may be based on criteria such as: iterations required, robustness in the presence of measurement noise, number of measurements needed, robustness with respect to knowledge of the inital conditions, and robustness with respect to initial assumptions regarding the form of the nonlinearity, depending on the needs of the particular application.

Among the methods which linearize the nonlinear system are those presented by Jedner and Unbehauen [4] and Ibanez [5]. Jedner and Unbehauen [4] represent a nonlinear system, which may often function at a number of operating points, by an equivalent number of linear submodels. It is assumed that the system operates at only a few points. Although the model is good for controller design, the point at which the system is operating must be known and the linear models apply only within the operating regions. Ibanez [5] takes a slightly different approach by assuming the system response to be periodic at the forcing frequency. An approximate transfer function is constructed. The 
tranfer function is dependent on the amplitude as well as on the exciting frequency and is valid only within the region of exciting frequencies.

The direct equation approach is used by Yasuda, Kawamura and Watanabe [6], [7]. The input and output measurements of a dynamic process are expressed in a Fourier Series using, for example, an FFT algorithm. The system nonlinearity is represented as a sum of polynomials with unknown coefficients. Applying the principle of harmonic balance, the polynomial coefficients as well as the other system parameters are obtained accurately. Knowledge of the nonlinearity is needed to construct the polynomial. Truncation in the Fourier Series expansion of the input or output may lead to error.

The regression estimation approach is used by Billings and Voon [8] and Greblick and Pawlak [9]. Billings and Voon [8] use the NARMAX model (Nonlinear Auto Regressive Moving Average model with eXogenous inputs) to represent the nonlinear system. A stepwise regression method determines the significant terms in the NARMAX model. Then a prediction-error algorithm provides optimal estimates of the final model parameters. Greblick and Pawlak [9] represent the linear dynamic submodel by an ARMA model and the nonlinearities by a Borel function. A non-parametric kernel regression estimation is employed to obtain the final analytical model.

Kortman and Unbehauen [10] and Distefano and Rath [11] use the minimization of an error cost function as a means of obtaining the coefficients of the functions used to represent the nonlinearities. The method presented by Kortman and Unbehauen [10] uses only system input and output information to estimate the polynomial representing the nonlinearities and the parameters of the linear components. It is robust in the presence of noise, although iteration is necessary. Distefano and Rath [11] present two techniques, a non-iterative direct identification and an iterative direct identification. In the first technique, measurement of all variables is required and the model parameters are obtained through the minimization of an error function. In the second technique, iteration is used to minimize a cost function yielding the system parameters in addition to the state trajectories. In Distefano and Rath [11], the nonlinear model form is also taken to be known.

In other techniques, as in statistical linearization, a nonlinear relation is replaced by a linear equivalent gain. Broersen [12] extends the technique of statistical linearization by representing the nonlinearity as a linear combination of a number of arbitrary functions. Correlation techniques are then used to determine the coefficients of these functions. The number and type of functions selected depends on the desired accuracy as well as some knowledge of the system nonlinearity. Reasonable accuracy is obtained in the presence of noise and no iterations are necessary. Although some of the basic properties of the true nonlinear output are preserved, it is limited to only random excitation, and knowledge of all states and forcing terms is required.

In the method of multiple scales (Hanagud, Mayyappa and Craig [13]), a perturbation solution to the nonlinear equation of motion is obtained. An objective function is built 
employing an integral least squares approach. The minimization of the functional yields the unknown parameters. Data on only one field variable is necessary, and the method is effective in the presence of high noise. The method of multiple scales, however, is restricted to systems with small damping and slight nonlinearities and, as in most other methods, the form of the nonlinearity is assumed a priori. The method typically requires some algebraic manipulations which may be quite involved, and these manipulations are only valid for a particular assumed nonlinear form. If the assumed nonlinear form is changed, the algebra must be repeated.

Another popular approach is to describe the nonlinear system using the Volterra or Wiener kernels. The Volterra series consists of the summation of impulse responses of increasing dimensionality. The Wiener series is also a set of orthogonal functions in which the input is white gaussian noise. Marmarelis and Udwadia [14], for example, estimate the first and higher order kernels appearing in the Volterra series using correlation techniques. Chen, Ishii and Suzumura [15] use cross-correlation functions in addition to the Volterra and Wiener series to describe nonlinear models and to show the relation between the system inner structure and the series. Although weakly nonlinear systems can be described by the first few kernels, for strongly nonlinear systems these series give accurate numerical results only at the expense of an excessive number of coefficients. This renders the analytical model impractical for control applications.

Other popular series are orthogonal polynomials such as Legendre (Wang and Chan [16]), Chebyshev, and Jacobi (Horn and Chou [17]). Horn and Chou [17] expand the variables of the system into a shifted Jacobi series, reducing the nonlinear state equation into a linear algebraic matrix equation. The unknown parameters of the nonlinear system are then estimated using least squares. Even though the algorithm works well in the presence of noise, the nonlinear form must be known a priori.

Another technique used for the identification of nonlinear systems is the extended Kalman filter. The extended Kalman filter is the linear Kalman filter applied to nonlinear systems by linearizing the nonlinear model into a Taylor series expansion about the estimated state vector. Yun and Shinozuka [18] apply the extended Kalman filter for the parameter estimation of a quadratic term. The state vector is augmented by including the unknown parameters in addition to the state variables. Through a series of iterations, the response, as well as the unknown parameters, are estimated by the Kalman filter. Among its disavantages are high sensitivity to initial conditions, in particular if the initial conditions are barely known.

Hammond, Lo and Seager-Smith [19] use an optimal control technique based on optimal control methods employed for linear system deconvolution. The form of the linear model is assumed to be known as well as the input and the output. A cost functional consisting of the weighted sum of the square of the error (between the actual and estimated output) yields an optimal estimated input. The estimated input and the actual input are 
used to obtain the nonlinearity as a function of the state variables. Although no previous assumption is made of the nonlinearities, there is no provision to deal with noise.

In previous papers, the Minimum Model Error algorithm (MME) was explained in detail (Mook and Junkins [20]), modified for nonlinear identification (Mook [21]), and shown to accurately identify exotic nonlinearities in higher order systems (Stry and Mook [22]). In this paper, it is shown how the MME algorithm successfully identifies nonlinearities using experimental data. An analytical model representing a harmonic oscillator with quadratic position feedback is studied. First, output data is obtained from a digital computer simulation of the nonlinear system and the quadratic term is identified to illustrate the accuracy of the technique on a known system. Second, an attempt is made to duplicate the nonlinear model using an analog computer. It is shown that despite the inability of the analog computer to produce a true quadratic term, the Minimum Model Error algorithm is capable of identifying a nonlinear model which accurately reproduces the analog output. The Minimum Model Error method produces a numerically stable identification regardless of the analog data initial conditions or record length.

\section{MME Algorithm}

In this section, we review the MME algorithm and how it is used to identify nonlinear dynamic systems. A more detailed explanation may be found in Mook and Junkins [20], Mook [21], and Stry and Mook [22].

The MME may be summarized as follows. Suppose there is a nonlinear system whose exact analytical representation is unknown, but for which output measurements are available. Using "normal" means (analysis, finite elements, etc.), a system model is constructed. As shown in [21]-[22], the MME will work well even if this system model is poor. The MME combines the assumed model with the measurements to determine the correct form of the nonlinear system. A correction term which represents the error in the model is added to the assumed model and a cost functional is formed. Minimization of the cost functional yields the model error. Subsequently, a least squares fit is performed on the error term to determine the correct form of the nonlinear system.

Consider a forced nonlinear dynamic system which may be modeled in state-space form by the equation

$$
\underline{\dot{x}}(t)=A \underline{x}(t)+\underline{F}(t)+\underline{f}(\underline{x}(t), \underline{\dot{x}}(t))
$$

where $\underline{x}(t)$ is the $n \times 1$ state vector consisting of the system states, $A$ is the $n \times n$ state matrix, $\underline{F}(t)$ is an $n \times 1$ vector of known external excitation, and $\underline{f}(\underline{x}(t), \underline{\dot{x}}(t))$ is an $n \times 1$ vector which includes all of the system nonlinearities. State-observable discrete time domain measurements are available for this system in the form

$$
\underline{\bar{y}}\left(t_{k}\right)=\underline{g}_{k}\left(\underline{x}\left(t_{k}\right), t_{k}\right)+\underline{v}_{k}, \quad t_{0} \leq t_{k} \leq t_{f}
$$


where $\underline{\bar{y}}\left(t_{k}\right)$ is an $m \times 1$ measurement vector at time $t_{k}, \underline{g}_{k}$ is the accurate model of the measurement process, and $\underline{v}_{k}$ represents measurement noise. $\underline{v}_{k}$ is assumed to be a zero-mean, gaussian distributed process of known covariance $R_{k}$. The measurement vector $\overline{\underline{y}}\left(t_{k}\right)$ may contain one or more of the system states. To implement MME, assume that a model, which is generally not the true system model because of the difficulties inherent in obtaining the true system model, is constructed in state-vector form as

$$
\underline{\dot{x}}(t)=A \underline{x}(t)+\underline{F}(t)
$$

Here, we show a linear model because in practice, linearization is the most common approach to modeling nonlinear systems. MME uses the assumed linear model in (3) and the noisy measurements in (2) to find the model error.

The model error, which includes the unknown nonlinear term(s) of the system, is represented by the addition of a correction term to the assumed linear model as

$$
\underline{\dot{x}}(t)=A \underline{x}(t)+\underline{F}(t)+\underline{d}(t)
$$

where $\underline{d}(t)$ is the $n \times 1$ correction term (dynamic model error) to be estimated later.

A cost functional, $J$, that consists of the weighted integral square of the correction term plus the weighted sum square of the measurement-minus-estimated measurement residuals, is formed:

$$
\begin{gathered}
J=\sum_{k=1}^{M}\left\{\left[\underline{\tilde{y}}\left(t_{k}\right)-\underline{g}_{k}\left(\hat{\underline{x}}\left(t_{k}\right), t_{k}\right)\right]^{T} R_{k}^{-1}\left[\underline{\tilde{y}}\left(t_{k}\right)-\underline{g}_{k}\left(\underline{\hat{x}}\left(t_{k}\right), t_{k}\right)\right]\right\} \\
+\int_{t_{o}}^{t_{j}} \underline{d}(\tau)^{T} W \underline{d}(\tau) d \tau
\end{gathered}
$$

where $M$ is the number of measurement times, $\underline{\hat{x}}\left(t_{k}\right)$ is the estimated state vector and $W$ is a weight matrix to be determined.

$J$ is minimized with respect to the correction term, $\underline{d}(t)$. The necessary conditions for the minimization lead to the following two point boundary value problem (TPBVP), (see Geering [23]),

$$
\begin{aligned}
\underline{\dot{x}}(t) & =A \underline{x}(t)+\underline{F}(t)+\underline{d}(t) \\
\underline{\dot{\lambda}}(t) & =-A^{T} \underline{\lambda}(t) \\
\underline{d}(t) & =-\frac{1}{2} W \underline{\lambda}(t) \\
\underline{\lambda}\left(t_{k}^{+}\right) & =\underline{\lambda}\left(t_{k}^{-}\right)+2 H_{k} R_{k}^{-1}\left[\underline{\tilde{y}}\left(t_{k}\right)-\underline{g}_{k}\left(\underline{\hat{x}}\left(t_{k}\right), t_{k}\right)\right] \\
H_{k} & =\left.\frac{\delta g}{\delta \underline{x}}\right|_{\underline{\hat{x}}\left(t_{k}\right), t_{k}} \\
\underline{x}\left(t_{o}\right) & =\underline{x}_{o} \quad \text { or } \quad \underline{\lambda}\left(t_{o}\right)=0 \\
\underline{x}\left(t_{f}\right) & =\underline{x}_{f} \quad \text { or } \quad \underline{\lambda}\left(t_{f}\right)=0
\end{aligned}
$$


where $\underline{\lambda}(t)$ is a vector of costates (Lagrange multipliers). Estimates of the states and of the dynamic model error are produced by the solution of this two-point boundary value problem. The estimates depend on the particular value of $W$. The solution is repeated until a value of $W$ is obtained which produces state estimates which satisfy the "covariance constraint", explained next.

According to the covariance constraint, the measurement-minus-estimated measurement residual covariance matrix must match the measurement-minus-truth error covariance matrix. This may be written as

$$
\left[\underline{\tilde{y}}\left(t_{k}\right)-\underline{g}_{k}\left(\underline{\hat{x}}\left(t_{k}\right), t_{k}\right)\right]^{T}\left[\underline{\bar{y}}\left(t_{k}\right)-\underline{g}_{k}\left(\underline{\hat{x}}\left(t_{k}\right), t_{k}\right)\right] \approx R_{k}
$$

During the minimization, the weight $W$ is varied until the state estimates satisfy the covariance constraint, i.e., the left hand side of Eq. (6) is approximately equal to the right hand side. The correction term or model error is, therefore, the minimum adjustment to the model required for the estimated states to predict the measurements with approximately the same covariance as the measurement error.

The TPBVP represented by Eqs. (5a) to (5f) contains jumps in the costates and, consequently, in the correction term. As evident from Eq. (5d), the size of the jump is directly proportional to the measurement residual at each measurement time. The noisier the measurements, the larger the jump size. A multiple shooting algorithm, developed by Mook and Lew [24], converts this jump-discontinuous TPBVP into a set of linear algebraic equations which may be solved using any linear equation solver. Multiple shooting also facilitates the analysis of a large number of measurements, by processing the solution at the end of every set of jumps.

After $W$ has been determined such that the state estimates satisfy the covariance constraint, the final step in the identification procedure is to use a least squares algorithm to fit the model error $d(t)$ to the unknown dynamic term(s). The error is expanded into some combination of linear and nonlinear terms, for example,

$$
d(t)=\alpha x(t)+\beta x^{2}(t)+\gamma x^{3}(t)+\ldots
$$

where $\alpha, \beta, \gamma, \ldots$ are unknown coefficients to be determined by least squares. Presumably, the least squares fit of Eq. (7) will find zero coefficients for the terms in the expansion which are not part of the true model, and nonzero coefficients for the actual model terms. Eq. (7) may be sampled repeatedly to obtain

$$
\begin{aligned}
& d\left(t_{1}\right)=\alpha x\left(t_{1}\right)+\beta x^{2}\left(t_{1}\right)+\gamma x^{3}\left(t_{1}\right)+\ldots \\
& d\left(t_{2}\right)=\alpha x\left(t_{2}\right)+\beta x^{2}\left(t_{2}\right)+\gamma x^{3}\left(t_{2}\right)+\ldots \\
& \vdots=\quad \vdots \\
& d\left(t_{l}\right)=\alpha x\left(t_{l}\right)+\beta x^{2}\left(t_{l}\right)+\gamma x^{3}\left(t_{l}\right)+\ldots
\end{aligned}
$$

or, in matrix form,

$$
\underline{D}_{l \times 1}=M_{l \times p} \underline{P}_{p \times 1}
$$


where $P=\left[\begin{array}{llll}\alpha & \beta & \gamma & \ldots\end{array}\right]^{T}$ is the vector of coefficients for the terms in $d(t)$. Since estimates of $d(t)$ are available continuously throughout the time domain, the parameter $l$ may be chosen quite large to improve the least squares fit. Generally, because of the jump discontinuities in the model error estimates at the measurement times, it is desirable to pick the least squares sampling times in Eq. (8) at points other than the measurement times. The least squares estimate is found by minimizing the following cost functional with respect to $P$ :

$$
\Phi=[\underline{D}-M \underline{P}]^{T}[\underline{D}-M \underline{P}]
$$

The solution is given by

$$
P=\left(M^{T} M\right)^{-1} M^{T} \underline{D}
$$

The multiple shooting algorithm presented by Mook and Lew [24] was used to obtain the MME solutions used in the tests presented in this paper. It was assumed in the examples that MME obtained the dynamic error term without knowledge of the boundary conditions on $\boldsymbol{E}$, so some distortion of the correction term at the initial and final times was expected due to the constraints of Eqs. (5e-5f), i.e., by assuming no state knowledge is available at $t_{0}$ or $t_{f}$, we constrain $\lambda\left(t_{0}\right)=0$ and $\lambda\left(t_{f}\right)=0$. Therefore, in all test cases, the initial and final ten percent of the correction term data was ignored in the least squares fit.

\section{Application Examples}

Two nonlinear equations of motion were studied, which represent the motion of an undamped harmonic oscillator with different amounts of quadratic position feedback (identical equations may arise in other physical systems as well). The equations in state space form are

$$
\begin{aligned}
& \left(\begin{array}{l}
\dot{x} \\
\dot{x}
\end{array}\right)=\left(\begin{array}{cc}
0 & 1 \\
-1 & 0
\end{array}\right)\left(\begin{array}{l}
x \\
\dot{x}
\end{array}\right)+\left(\begin{array}{c}
0 \\
-0.526 x^{2}
\end{array}\right) \\
& \left(\begin{array}{l}
\dot{x} \\
\dot{x}
\end{array}\right)=\left(\begin{array}{cc}
0 & 1 \\
-1 & 0
\end{array}\right)\left(\begin{array}{l}
x \\
\dot{x}
\end{array}\right)+\left(\begin{array}{c}
0 \\
-1.137 x^{2}
\end{array}\right)
\end{aligned}
$$

where $x$ is position, and the dot indicates differentiation with respect to time. No forcing was applied.

In the following discussion, Eq. (11) is denoted Model A and Eq. (12) is denoted Model B. Different initial conditions were used for each system, for a total of five different tests. These are shown in Table 1. 
Table 1. List of conditions used for each test

\begin{tabular}{|c|c|c|c|}
\hline Test \# & $\boldsymbol{x}\left(t_{0}\right)$ & $\dot{x}\left(t_{0}\right)$ & $x^{2}$ \\
\hline $\mathrm{A} 1$ & 0.00 & 0.08 & -0.526 \\
\hline $\mathrm{A} 2$ & 0.00 & 0.26 & -0.526 \\
\hline $\mathrm{A} 3$ & 0.00 & 0.52 & -0.526 \\
\hline $\mathrm{B} 1$ & 0.00 & 0.08 & -1.137 \\
\hline $\mathrm{B} 2$ & 0.00 & 0.26 & -1.137 \\
\hline
\end{tabular}

To utilize MME, the linear part of Eqs. (11) and (12) was assumed to be known, rendering the model error equivalent to the nonlinear term, $c * x^{2}$. Data for the MME nonlinear identification was generated from two different sources. First, noiseless position measurements were gathered from a digital computer simulation for all five tests. Application of MME to the measurements yielded an accurate estimate of the nonlinear term in each case. Then, an attempt was made to duplicate each system on an analog computer. Even though the analog computer did not reliably reproduce the quadratic term, the position measurements for all five tests were recorded and nonlinear models identified. MME proved capable of identifying accurate nonlinear models for the analog output.

\section{Digital computer simulation results}

One hundred noiseless position measurements were generated on a VAX 780 for the five test cases shown in Table 1.. A sampling rate of $4 \mathrm{Hertz}$ was used. Three terms were employed in the least squares fit: $x, \dot{x}$ and $x^{2}$. The resulting numerical values are shown in Table 2.

Table 2. Least Squares estimates of the dynamic model error employing analytically generated measurements in the MME algorithm.

\begin{tabular}{|c|c|c|c|c|c|c|}
\hline $\begin{array}{c}\text { Test } \\
\#\end{array}$ & MME & MME & MME & Analytic & Analytic & Analytic \\
\hline A1 & $4.24 \times 10^{-4}$ & $4.90 \times 10^{-4}$ & -0.526 & 0.0 & 0.0 & -0.526 \\
\hline A2 & $1.21 \times 10^{-3}$ & $-3.00 \times 10^{-4}$ & -0.527 & 0.0 & 0.0 & -0.526 \\
\hline A3 & $2.49 \times 10^{-3}$ & $2.35 \times 10^{-3}$ & -0.528 & 0.0 & 0.0 & -0.526 \\
\hline B1 & $-2.86 \times 10^{-4}$ & $-6.39 \times 10^{-3}$ & -1.138 & 0.0 & 0.0 & -1.137 \\
\hline B2 & $-3.46 \times 10^{-4}$ & $-6.80 \times 10^{-3}$ & -1.138 & 0.0 & 0.0 & -1.137 \\
\hline
\end{tabular}

MME identifies the quadratic term with great accuracy in all five tests. A plot of the estimated, analytical and measured position is shown in Figure (1a) for test case A1. 
Figure (1b) presents the predicted model error and the dynamic model error estimated by MME for test case A1. MME estimates the position and correction term with great accuracy. Similar results are obtained for tests A2, A3, B1, and B2, but are not shown.

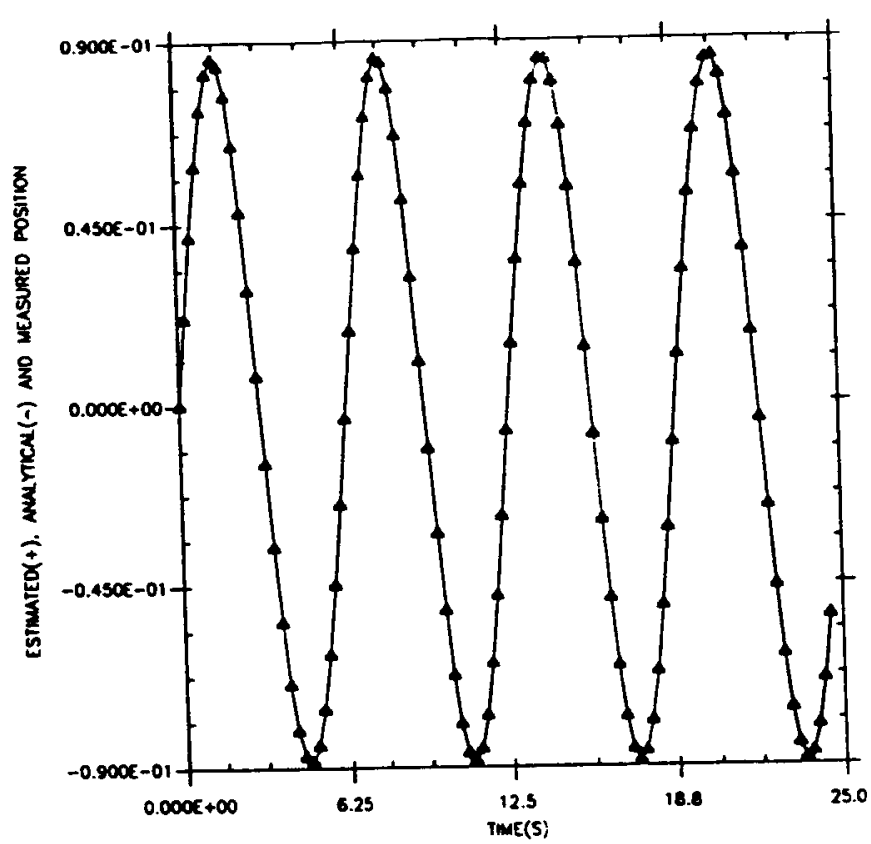

(a)

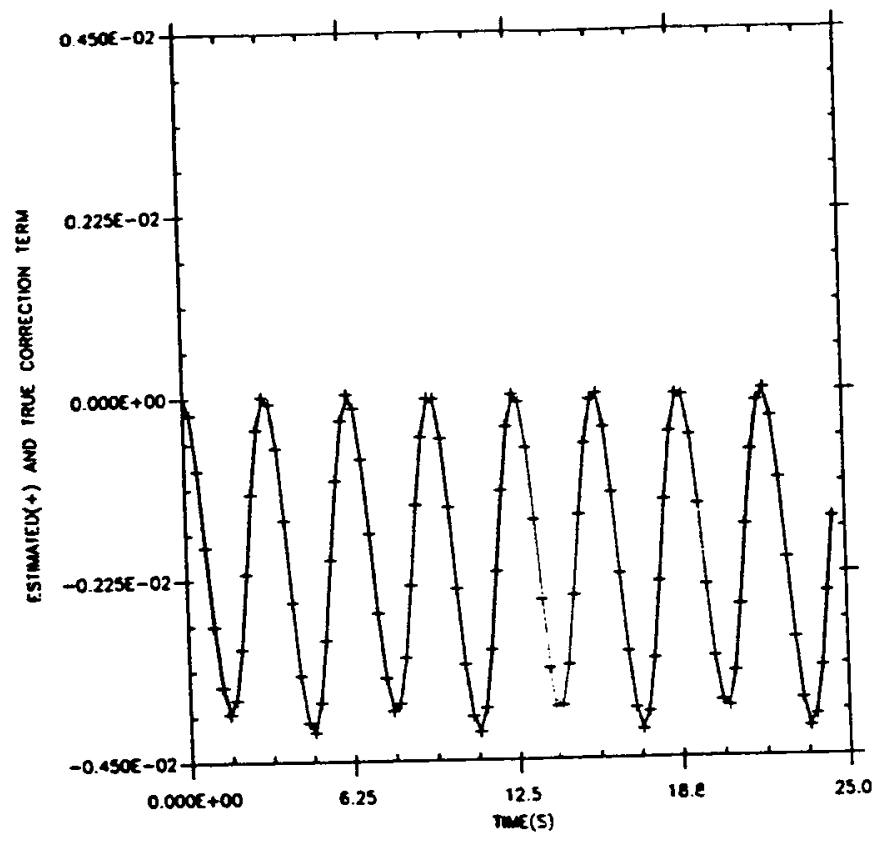

(b)

Figure 1 (a) Analytical, measured ( $\Delta$ ), and MME estimated position for test A1 using digital computer simulated measurements. The analytical and MME estimates are essentially identical (solid line).

(b) MME estimate (+) and actual model error.

\section{Analog computer results}

Three hundred fifty position measurements were generated on a Comdyna GP-6 analog computer for all five test cases. One hundred measurements with a sampling rate of $4 \mathrm{Hertz}$ were used in the analysis. The identification procedure yielded the numerical values shown in Table 3. 
Table 3. Least Square estimates of the nonlinear terms using measurements generated by the analog computer.

\begin{tabular}{|c|c|c|c|}
\hline $\begin{array}{c}\text { Test } \\
\#\end{array}$ & $\begin{array}{c}\text { MME } \\
x\end{array}$ & $\begin{array}{c}\text { MME } \\
\dot{x}\end{array}$ & $\begin{array}{c}\text { MME } \\
x^{2}\end{array}$ \\
\hline A1 & -0.20 & $1.04 \times 10^{-2}$ & -6.17 \\
\hline A2 & $-5.06 \times 10^{-2}$ & $-1.89 \times 10^{-3}$ & -1.322 \\
\hline A3 & $-6.44 \times 10^{-3}$ & $-4.81 \times 10^{-3}$ & -0.689 \\
\hline B1 & 0.10 & $-3.78 \times 10^{-3}$ & -3.47 \\
\hline B2 & $2.55 \times 10^{-2}$ & $7.42 \times 10^{-3}$ & -1.265 \\
\hline
\end{tabular}

The numerical results for the least squares fit of the error term did not match the analytically predicted coefficients. The reason for the numerical discrepancy was the analog's failure to produce a dependable quadratic term. Table 4 shows some position values squared by the analog. The analog consistently produced an error in the quadratic term. The recorded data, although containing errors due to quadratic term, is believed to be practically noiseless.

Table 4. Quadratic term produced by the analog computer.

\begin{tabular}{|c|c|c|}
\hline$x$ & $x^{2}$ & Analog \\
\hline 2.00 & 4.00 & 4.30 \\
\hline 2.50 & 6.25 & 7.00 \\
\hline 3.00 & 9.00 & 9.50 \\
\hline
\end{tabular}

Figures (2)-(6) show the analytical position, analog measurements and position predicted by the MME analysis for all analog tests. The MME identification produced good state estimates and a model which matched the measured data much better than the analytical models in Eqs. (11) and (12).

Note, these results were obtained without knowledge of the initial or final state vector value. As shown in Eqs. (5e) and (5f), by setting the initial and final costate values to zero, no knowledge of the initial or end conditions are necessary. Also, the same results presented in Table 3 were obtained when using all three hundred and fifty measurements instead of one hundred measurements in the MME algorithm. 


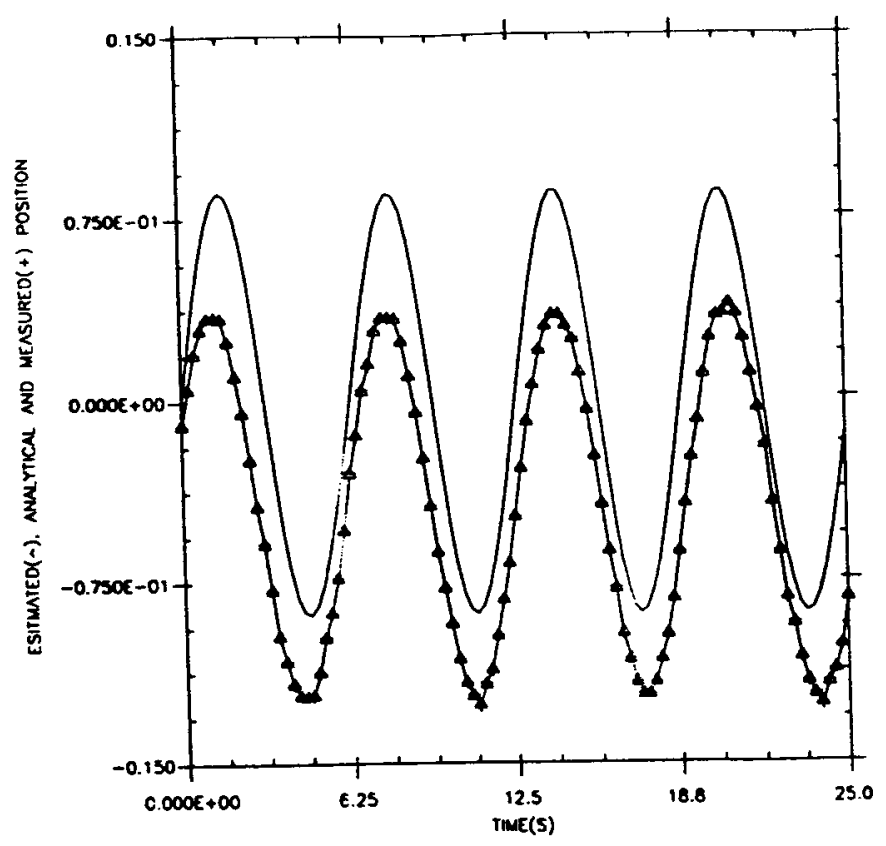

Figure 2 Analytical, measured $(\Delta)$, and MME estimated position for test A1 using analog computer measurements. The MME estimates are essentially identical to the measurements

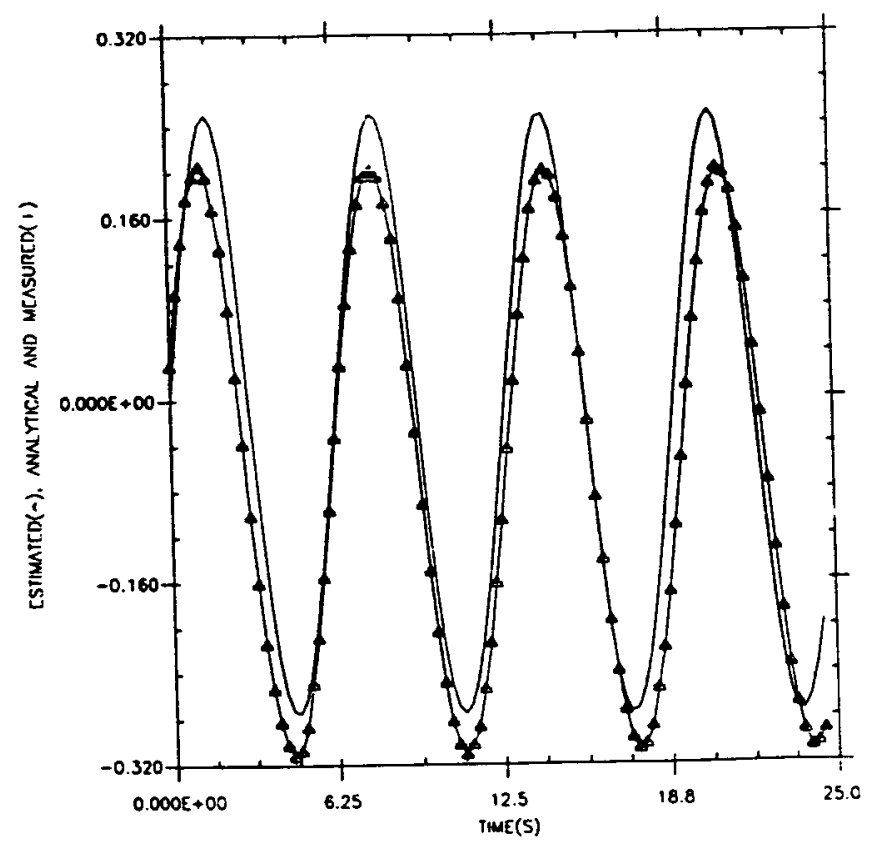

Figure 3 Analytical, measured $(\Delta)$, and MME estimated position for test A2 using analog computer measurements. The MME estimates are essentially identical to the measurements 


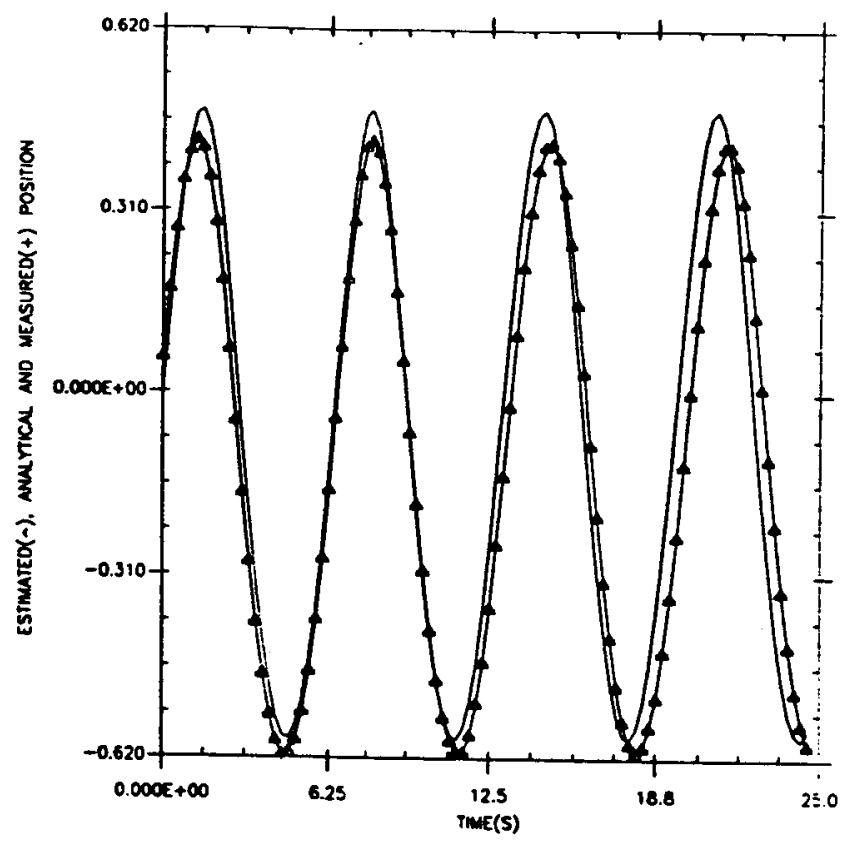

Figure 4 Analytical, measured $(\Delta)$, and MME estimated position

for test A3 using analog computer measurements. The MME estimates are essentially identical to the measurements

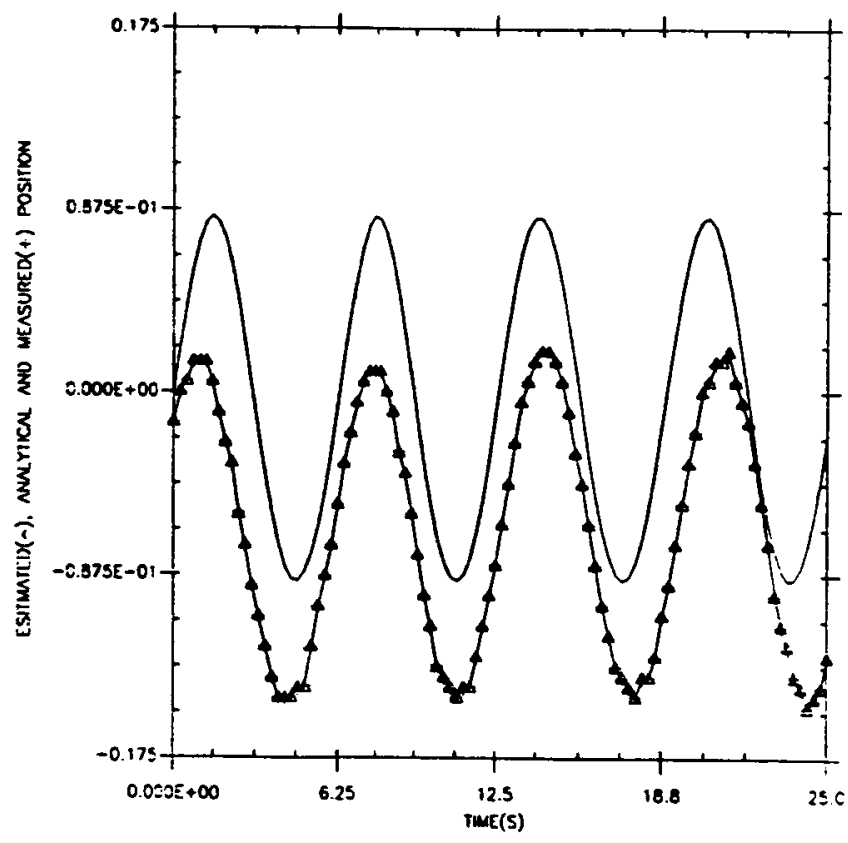

Figure 5 Analytical, measured $(\Delta)$, and MME estimated position for test B1 using analog computer measurements. The MME estimates are essentially identical to the measurements 


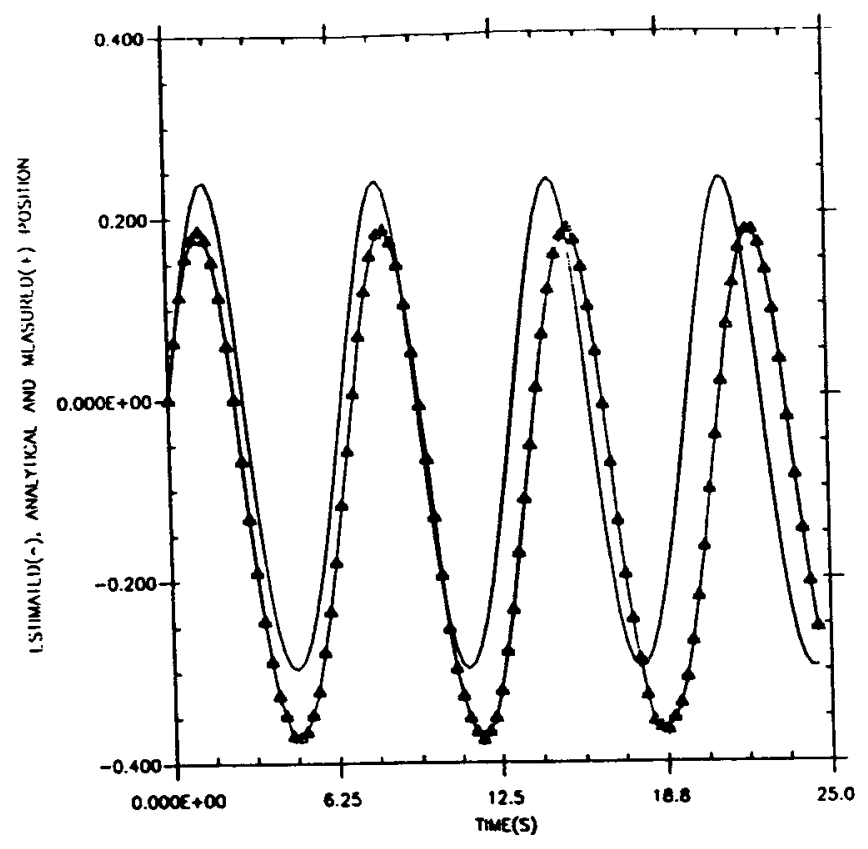

Figure 6 Analytical, measured $(\Delta)$, and MME estimated position for test B2 using analog computer measurements. The MME estimates are essentially identical to the measurements

\section{Conclusion}

In this paper, an MME-based algorithm was used to identify the quadratic term of a nonlinear harmonic oscillator. For demonstration purposes, data was obtained from two sources. Output data obtained from a digital computer simulation was used to verify the accuracy of the method. Then, data from an analog computer was used as a test of the method on "real" data. It was shown that despite the inability of the analog computer to reproduce a true quadratic term, the MME algorithm was capable of identifying a nonlinear model which accurately reproduced the analog output. This result indicates that the method is robust with respect to (lack of) a priori knowledge of the system dynamics. The identification was accurate regardless of initial conditions or data record length, indicating that the method is also robust with respect to those variables.

\section{References}

1. Thompson, J.M.T., and Stewart, H.B., Nonlinear Dynamics and Chaos, Wiley, New York, 1986.

2. Nayfeh, A. H., and Mook, D. T., Nonlinear Oscillations, Wiley, New York, 1979. 
3. Natke, H.G., Juang, J.-N., and Gawronski, W., "Identification of Nonlinear Mechanical Systems: A Brief Review," NASA Langley Research Center, Hampton, VA, U.S.A. .

4. Jedner, U., and Unbehauen, H., "Identification of a Class of Nonlinear Systems by Parameter Estimation of a Linear Multi-Model," IMACS, Modeling and Simulation for Control of Lumped and Distributed Parameter Systems, June 3-6, 1986, pp. 11-15.

5. Ibanez, P., "Identification of Dynamic Parameters of Linear and Non-Linear Structural Models from Experimental Data," Nuclear Engineering and Design, Vol. 25, 1973, pp. $32-41$.

6. Yasuda, K., Kawamura, S., and Watanabe, K., "Identification of Nonlinear MultiDegree-of-Freedom Systems (Identification Under Noisy Measurements)," JSME International Journal, Vol. 3, No. 1, 1988, pp. 502-509.

7. Yasuda, K., Kawamura, S., and Watanabe, K., "Identification of Nonlinear MultiDegree-of-Freedom Systems (Presentation of an Identification technique)," JSME International Journal, Vol. 3, No. 1, 1988, pp. 8-14.

8. Billings, S.A., and Voon, W.S.F., "A Prediction-Error and Stepwise Regression Estimation Algorithm for Nonlinear Systems," Int. J. Control, Vol. 44, No. 3, 1986, pp. 803-822.

9. Greblick, W., and Pawlak, M., "Hammerstein System Identification by NonParametric Regression Estimation," Int. J. Control, Vol. 45, No. 1, 1987, pp. 343-354.

10. Kortmann, M., and Unbehauen, H., "Application of a Recursive Prediction Error Method to the Identification of Nonlinear Systems Using the Weiner Model," IMACS, Modeling and Simulation for Control of Lumped and Distributed Parameter Systems, June 3-6, 1986, pp. 3-9.

11. Distefano, N., and Rath, A., "System Identification in Nonlinear Seismic Dynamics," Computer Methods in Applied Mechanics and Engineering, Vol. 5, 1975, pp. 353-372.

12. Broersen, P.M.T., "Estimation of Parameters of Non-Linear Dynamical Systems," Int. J. Non-Linear Mechanics, Vol. 9, 1974, pp. 355-361.

13. Hanagud, S.V., Meyyappa, M., and Craig, J.I., "Method of Multiple Scales and Identification of Nonlinear Dynamical Systems," AAIA J., Vol. 23, No. 5, 1985, pp. 802-807.

14. Marmarelis, P.Z., and Udwadia, F.E., "The Identification of Building Structural Systems-PartII: The Nonlinear Case," Bulletin of the Seismological Society of America, Vol. 66, 1979, pp. 153-171.

15. Chen, H., Ishii, N., and Suzumura, N., "Structural Classification of Non-Linear Sytems by Input and Output," Int. J. Systems Sci., Vol. 17, No. 5, 1986, pp. 741-774.

16. Wang, M.L., and Chang, R.Y., "Model Reduction and Control System Design by Shifted Legendre Polynomial Functions," A.S.M.E. J. Dynam. Sys. Meas. Control, 
Vol. 105, 1983, pp. 52-55.

17. Horn, I-R., and Chou, J-H., "Analysis and Identification of Non-Linear Systems via Shifted Jacobi Series," Int. J. Control, Vol. 45, No. 1, 1987, pp. 279-290.

18. Yun, C-B., and Shinozuka, M., "Identification of Nonlinear Structural Dynamic Systems," J. Struct. Mech., Vol. 8, No. 2, 1980, pp. 187-203.

19. Hammond, J.K., Lo, H.R., and Seager-Smith, E.J., "Identification of Nonlinearities in Vibrating Systems Using Optimal Control Techniques," IMAC, 1987, pp. 1467-1473.

20. Mook, D.J., and Junkins, "Minimum Model Error Estimation for Poorly Modeled Dynamic Systems", AIAA Journal of Guidance, Control, and Dynamics, Vol. 11, No. 3, 1988, pp. 256-261.

21. Mook, D.J., "Estimation and Identification of Nonlinear Dynamic Systems," AIAA J., Vol. 27, No. 7, 1989, pp. 968-974.

22. Stry, G.I., and Mook, D.J., "Identification of Higher-Order Systems with Exotic Nonlinearities," in review.

23. Geering, H.P., "Continuous Time Optimal Control Theory for Cost Functionals Including Discrete State Penalty Terms," IEEE Trans. A.C., Vol. AC-21, 1976, pp. 866-869.

24. Mook, D.J., and Lew, J-H., "Multiple Shooting Algorithm for Jump Discontinuous Problems in Optimal Control and Estimation," to appear in the IEEE Trans. A. C. . 